Surveillance of primary sclerosing cholangitis with $\mathrm{ERC}$ and brush cytology : risk factors for cholangiocarcinoma

\title{
Boyd, Sonja
}

2017

Boyd , S , Mustonen , H , Tenca , A , Jokelainen , K, Arola , J \& Farkkila , M A 2017 , ' Surveillance of primary sclerosing cholangitis with ERC and brush cytology : risk factors for cholangiocarcinoma ' , Scandinavian Journal of Gastroenterology , vol. 52 , no. 2 , pp. 242-249 . https://doi.org/10.1080/00365521.2016.1250281

http://hdl.handle.net/10138/311698

https://doi.org/10.1080/00365521.2016.1250281

cc_by_nc_nd

acceptedVersion

Downloaded from Helda, University of Helsinki institutional repository.

This is an electronic reprint of the original article.

This reprint may differ from the original in pagination and typographic detail.

Please cite the original version. 
Surveillance of Primary Sclerosing Cholangitis with ERC and Brush Cytology: risk factors for cholangiocarcinoma

Short title: Surveillance of PSC

Boyd Sonja ${ }^{1}$, Mustonen Harri², Tenca Andrea ${ }^{3}$, Jokelainen Kalle ${ }^{3}$, Arola Johanna ${ }^{1}$, Färkkilä Martii $\mathrm{A}^{3}$

${ }^{1}$ Department of Pathology, University of Helsinki, and Helsinki University Hospital, Helsinki, Finland

${ }^{2}$ University of Helsinki and Department of Surgery, Helsinki University Hospital, Helsinki, Finland

${ }^{3}$ University of Helsinki and Clinic of Gastroenterology, Helsinki University Hospital, Helsinki, Finland

Corresponding author: Sonja Boyd, Meilahti Laboratories of Pathology, Helsinki University Hospital, PO Box 400, 00029 HUS, Finland. E-mail: sonja.boyd@hus.fi; Tel: +358-50-4270551; Fax: +358-94-717-5372 


\section{Abstract}

Objective. Primary sclerosing cholangitis (PSC) is a chronic cholestatic liver disease leading to bile duct strictures and fibrosis, and predisposing to cholangiocarcinoma (CCA). Biliary dysplasia is a known precursor of CCA. In our unit PSC patients undergo regular surveillance with $\mathrm{ERC}$ and brush cytology $(\mathrm{BC})$, and liver transplantation is an option in case with biliary dysplasia. We evaluated the risk factors for biliary dysplasia and CCA based on ERC imaging, BC and liver function tests.

Patients and methods: 788 ERCs were performed with BC for 447 PSC patients. ERC images were evaluated using the modified Amsterdam score, neutrophilic inflammation was assessed in BC, and liver function tests were collected. Ploidy analysis with DNA flow cytometry was performed in cases with advanced PSC or previous suspicious $\mathrm{BC} /$ aneuploidy. The endpoint was either a benign disease course (follow-up for $\geq 2.4$ years after the latest ERC), benign histology, biliary dysplasia, or CCA.

Results: Benign disease course was seen in 424/447 (including 23 cases with biliary dysplasia), and CCA in 17 (3.8\%) patients. Gallbladder carcinoma/carcinoma in situ was diagnosed in 3 patients. Advanced ERC findings, male gender, suspicious BC, aneuploidy in flow cytometry, inflammation, and elevation of ALP, bilirubin, ALT, AST, GGT, CEA, and CA19-9 represented significant risk factors for CCA in univariate analysis.

Conclusions: PSC patients with advanced bile duct disease and elevated liver enzymes, CEA or CA19-9, inflammation or suspicious BC are most likely to develop CCA. These patients may benefit from surveillance with $\mathrm{BC}$ if early liver transplantation is possible.

Keywords: biliary dysplasia; cholangiocarcinoma; liver transplantation

Abbreviations: ALP, alkaline phosphatase; ALT, alanine aminotransferase; AST, aspartate aminotransferase; BC, brush cytology; CA19-9, carbohydrate antigen 19-9; CCA, 
cholangiocarcinoma; CEA, carcinoembryonic antigen; ERC, endoscopic retrograde cholangiography; GGT, gamma-glutamyl transferase; HR, hazard ratio; IBD, inflammatory bowel disease; LT, liver transplantation; mERC score, modified Amsterdam ERC score; PSC, primary sclerosing cholangitis; UDCA, ursodeoxycholic acid 
Primary sclerosing cholangitis (PSC) is a chronic progressive disease leading to strictures of the bile ducts and, eventually, cirrhosis. PSC patients carry a markedly increased risk for cholangiocarcinoma (CCA), with the lifetime risk reaching nearly $10 \%$ (1), or 398 -fold compared with the general population (2). CCA represents a common cause of death among PSC patients, whereby half of all CCAs are detected within one year of a PSC diagnosis $(3,4)$, depending on the indications for first endoscopic retrograde cholangiography (ERC) and magnetic resonance imaging. Several risk factors for CCA in PSC patients have been identified, such as a long history of inflammatory bowel disease (IBD), hyperbilirubinemia, and an older age at PSC diagnosis $(2,5)$. Like many other carcinomas, CCA is thought to develop through metaplasia, low-grade dysplasia, and high-grade dysplasia (6). CCA generally presents as a contraindication for liver transplantation (LT) and carries a dismal prognosis.

The diagnosis of early CCA is difficult with currently available methods. At present, no recommendations exist for the screening of PSC patients for biliary neoplasia without dominant strictures (7). In some centres, such as in our hospital, early LT to prevent dysplasia progression to CCA is an option if biliary dysplasia is repeatedly documented in brush cytology (BC) $(8,9)$. Only $30 \%$ of US transplantation centres screen for hepatobiliary malignancy with BC prior to LT (10), even among listed patients. BC possibly combined with other techniques is used to diagnose cytological dysplasia or CCA among PSC patients (8). $\mathrm{BC}$ is quite specific but its sensitivity is rather low (11). Inflammation of the bile ducts is thought to predispose for CCA (12). Inflammation may also induce atypia in the biliary epithelium, making BC interpretation challenging. Polysomy with fluorescence in situ hybridisation (13) or aneuploidy with DNA flow cytometry $(9,14)$ appear to increase the sensitivity and specificity for detecting biliary neoplasia. Most CCAs among PSC patients are located in the extrahepatic or perihilar bile ducts remaining reachable with $\mathrm{BC}(4)$. 
In our earlier study, we found that even in a mostly asymptomatic PSC population, advanced extrahepatic ERC changes and elevated aminotransferases during the diagnostic ERC represented significant risk factors for biliary neoplasia (15).

This study aimed to find patients at highest risk for CCA by using regular systematic surveillance with ERC with BC in a non-selected group of PSC patients, and to identify the risk factors associated with the development of CCA and dysplasia using detailed ERC scoring, $\mathrm{BC}$, and laboratory findings.

\section{Patients and methods}

This large, single-centre study in a population of PSC patients took place at Helsinki University Hospital, where ERC with BC were performed during the study period. Since 2006, PSC patients in our hospital regularly underwent ERC with BC, in addition to laboratory testing and imaging. The frequency of ERC depends on both the severity of the PSC (defined by ERC changes) and the previous BC findings (Figure 1).

Patients. We collected patient data from the Department of Pathology, Meilahti Laboratories, Helsinki University Hospital and the PSC registry of the Clinic of Gastroenterology, Helsinki University Hospital. We included PSC patients over 18 years undergoing ERC and BC surveillance between 1 January 2006 and 31 December 2011 in our study. In total, our sample included 460 PSC patients. Thirteen patients were excluded from the study due to loss of follow up (moved elsewhere or noncompliance). We also excluded patients with secondary sclerosing cholangitis and immunoglobulin G4 related cholangitis. Table I shows the patient characteristics. More than half of all patients (247 or $55 \%)$ were male. In our sample, 185 patients were diagnosed with PSC earlier than 2006 or elsewhere after 2006 and subsequently monitored in our unit (earlier cohort), and 262 were diagnosed with PSC 
in our unit between 2006 and 2011 (surveillance cohort). In total, 788 ERCs were performed (1-6 ERCs/patient). In 137 patients, only one ERC was performed, and among twenty-one of them it was due to reached endpoint (six CCAs, fifteen liver transplantations), rest of them not yet having the second ERC during surveillance time. In majority of the patients $(n=310)$, 2-6 ERCs were performed. ERCs were planned according to the surveillance protocol (Fig. 1). The median time between the first and the second ERC was 2.2 years, and thereafter 1.1 years. In $93 \%$ of the cases, the main indication for ERC was either verification of PSC diagnosis or surveillance according to protocol. In $6 \%$, the indication was evaluation for liver transplantation, and in $1 \%$, treating a stricture. However, strictures were treated with dilatation mostly during normal surveillance ERCs as indicated. The median age at the time of PSC diagnosis was 36 years. Most patients (74\%) presented with concomitant IBD, most commonly ulcerative colitis. We retrieved the presence of possible symptoms of liver disease (abdominal pain, jaundice, pruritus, cholangitis, fever, and fatigue) from patient charts. Only $18 \%$ of patients were symptomatic in the beginning of surveillance. We also registered the data on the use of ursodeoxycholic acid (UDCA).

ERCs. ERCs were performed by two experienced gastroenterologists (MF and $\mathrm{KJ}$ ) using the balloon occlusion technique to ensure adequate and standardised filling of intra- and extrahepatic bile ducts in successive ERCs. Images were evaluated using the modified Amsterdam score (mERC score) (15). BC was collected systematically in every patient from extra- and intrahepatic bile ducts, with the aim of reaching as many intrahepatic branches as possible, regardless of the presence or absence of dominant strictures, in order to collect a substantial number of cells for evaluation as described elsewhere (9).

Brush cytology. BCs were processed and evaluated as described earlier (9). We performed cytological evaluation from cytospin and cell-block slides using three-tiered criteria as benign, suspicious, or malignant (corresponding CCA). Suspicious cytology included any of 
the following features: biphasic cell population, lack of polarity, nuclear moulding, nuclear membrane irregularities, high nuclear-cytoplasm ratio, hyper/hypochromacy, and lack of cohesion. Neutrophilic inflammation in BC was evaluated semi-quantitatively $(0=$ neutrophils/epithelial cells $<0.05, \quad 1=$ neutrophils/epithelial cells $0.05-0.4$, and $2=$ neutrophils/epithelial cells $>0.4$ ). If the number of epithelial cells was low (below 200), we assumed that inflammation was absent.

DNA ploidity. In cases with advanced PSC or with a previous suspicious cytology or aneuploidy, we also collected a sample for DNA ploidy analysis. In these cases, a separate brush sample for DNA flow cytometry was collected and processed as previously reported (9). In this study, we considered a DNA index over 1.1 as aneuploid to avoid false-positive results.

Histology. Two pathologists (JA and SB) blinded to the original findings re-evaluated explanted livers. Bile ducts were graded as benign (including regenerative/inflammatory atypia), dysplastic (two-tiered evaluation to low-grade and high-grade; carcinoma in situ included in high-grade dysplasia), or invasive CCA. For patients with CCA and no LT, CCA was verified from histology, either from a surgical specimen or a core needle biopsy, or malignant BC with the imaging results consistent with CCA. Gallbladder carcinomas were verified from surgical specimens.

Laboratory values. Routine laboratory tests were taken the day before ERC using the appropriate methods. Results at the beginning of surveillance are presented in Table II.

Endpoints. The endpoints in the primary analyses of this study included 1) CCA, or 2) nonmalignant disease course with benign biliary histology or biliary dysplasia in an explanted liver or benign follow-up for at least 2.4 years following the last ERC. The status of patients with benign follow-up was monitored until 31 May 2014, and, for patients with LT or CCA, 
until LT was performed or CCA detected. Gallbladder carcinomas were only reported and not used as the endpoint, because the $\mathrm{BC}$ findings most likely did not provide information about gallbladder epithelium.

Statistics. Statistical analyses were performed using IBM SPSS statistical package version 22 (IBM, New York, NY, USA), SAS version 9.3 (SAS Institute Inc., Cary, NC, USA), Statexact v4 (Cytel Software Corporation, USA), R version 3.2.2 (The R foundation, Vienna, Austria) or with Medcalc version 14 (Medcalc Software, Ostend, Belgium). The MannWhitney $U$ test was used to evaluate continuous variables. For dichotomous variables, Fisher's exact test was used to compare differences between groups. Cox proportional hazards regression analysis was used for uni- and multivariate time to event analyses for dichotomous endpoints (CCA or dysplasia/benign histology/follow-up). The penalized maximum likelihood (lasso) with standardized variables (the glmnet R-package; www.cran.rproject.org) was also used. Inflammation in the BCs was calculated as inflammatory burden during the follow-up period, integrating the semi-quantitative neutrophil score $(0-2)$ over time to obtain the area under the curve. The result was normalised using the follow-up period, resulting in an average weighted score for inflammation ranging from 0 to 2 . A corresponding 'burden value' was also calculated for aspartate aminotransferase (AST), alanine aminotransferase (ALT), alkaline phosphatase (ALP), gamma glutamyl transferase (GGT), bilirubin, carcinoembryonic antigen (CEA), and carbohydrate antigen 19-9 (CA19-9) in addition to using the actual laboratory values. mERC score burden was also calculated from total, intrahepatic and extrahepatic mERC scores, correspondingly. For continuous variables, we used a logarithmic transformation if necessary. For comparison, a corresponding analysis was also performed using biliary neoplasia (CCA or dysplasia) or benign follow-up/histology as dichotomous endpoints (see Supplementary Table I). The first 
ERC date in our unit in the period beginning in 2006 was defined as the beginning of the surveillance period.

Ethics. The local ethics committee of Helsinki University Hospital for Internal Medicine, has approved the study protocol, number 278/13/03/01/2009.

\section{Results}

Endpoints. The median follow-up time was 6 years. In total, CCA was diagnosed in 17 (3.8\%) patients, including two with liver transplantation. The median time to diagnosing CCA after PSC diagnosis stood at 3 years (IQR $0.25-12.9$, range $0-19.5$ years). Two patients were diagnosed with CCA in the initial ERC, and three were diagnosed with CCA within five months of the initial ERC. Ten CCAs were located in extrahepatic, hilar or perihilar bile ducts, and three were intrahepatic. In four cases, the origin of the tumour could not reliably be determined. More detailed information about CCA patients is presented in Supplementary Table II. A majority (424 or $94.9 \%$ ) of patients presented with a non-malignant disease course, including biliary dysplasia in 23 ( 8 high-grade, 15 low-grade) explanted livers, benign biliary histology in 21 explanted livers and 383 patients with benign follow-up. LT was performed altogether for 46 (10.3\%) patients. Three patients had hepatocellular carcinoma in the explanted liver, two of them having also low-grade dysplasia of the bile ducts. After median 4.2 years of liver transplantation, 20/23 patients with biliary dysplasia of the explanted liver were alive with no signs of biliary malignancy. One patient had been diagnosed with adenocarcinoma of the papilla Vater. One died of malignancy in pancreas, and one of metastatic hepatocellular carcinoma. Gallbladder carcinoma or carcinoma in situ was diagnosed in three patients. The outcome of the surveillance cohort is presented in Figure 2. 
ERC scores. In the first ERC of this study period, we detected intrahepatic PSC alone in 256 patients, and intra- and extrahepatic disease in 191 patients (Table I). All patients with CCA during follow-up had both intra- and extrahepatic ERC changes in their first ERC.

Laboratory values. ALP measured at the beginning of surveillance was significantly higher among patients who developed CCA, as well as bilirubin, GGT, AST, ALT, CA19-9, and CEA $(p=<0.001-0.021)$. Table II shows the laboratory values at the beginning of the surveillance according to the endpoint. CA19-9 was over the upper limit of normal (ULN) ( $\geq 26 \mathrm{kU} / \mathrm{L})$ at some point during surveillance among 54 patients (12.1\%), 7 of whom were diagnosed with CCA and 5 with biliary dysplasia. CA19-9 was markedly elevated (>129 $\mathrm{kU} / \mathrm{L}$ ) among 7 patients, 5 of whom had CCA and 2 had a benign follow-up (16). On the other hand, CA19-9 was at some point elevated during surveillance in 7/17 (41.2\%) CCA patients and in $5 / 23(21.7 \%)$ of the patients with biliary dysplasia.

BCs and ploidy analysis. Among all 788 BCs, 713 (90.5\%) were benign, 72 (9.1\%) suspicious, and $3(0.4 \%)$ were malignant. Ploidy analysis was performed in 486 ERCs (62\%), out of which $28(5.8 \%)$ were aneuploid (DNA index $>1.1)$. BC was suspicious or malignant at least once in $12(70.6 \%)$ out of 17 CCA patients (Table III). Among the 21 explanted livers with benign biliary histology, 17 (81.0\%) showed preoperative benign and diploid BCs. In total, 185 patients presented with an inflammatory burden $>0.25$ in BC, with most CCAs (16/17) detected among them. If the inflammatory burden was low at $\leq 0.25$, only one CCA was detected among 262 patients.

Sensitivity and specificity. If one suspicious or malignant BC was regarded as a positive cytology and if all BCs must be benign for a negative cytology, the sensitivity to detect CCA with BC reached $71 \%$, with $91 \%$ specificity across the entire study population (Table III). There was a tendency toward better sensitivity in the surveillance cohort (100\%). Combining DNA flow cytometry and BC, the sensitivity and specificity were not significantly higher. If 
biliary neoplasia (CCA or dysplasia) was used as an endpoint, the sensitivity of BC was $65 \%$ with $94 \%$ specificity, not being significantly better combined with DNA flow cytometry. We found eight patients with DNA index of 1.50 or higher. Three of them were diagnosed with CCA and four had high-grade dysplasia.

Risk factors. Patients with more advanced biliary disease (mERC score burden $>8$ ) exhibited a significantly elevated hazard ratio $(\mathrm{HR})$ for CCA $(p<0.001$; Table IV). These results were similar for intrahepatic, extrahepatic and total mERC scores. Men exhibited a significantly higher HR for CCA compared with women $(p=0.025)$. Other significant factors for CCA in the univariate analysis included the inflammation burden in BC, ALT burden, AST burden, ALP burden, GGT burden, bilirubin burden, CEA > ULN burden and CA19-9 > ULN burden $(p<0.001)$. Furthermore, the burden of suspicious BCs or suspicious BC in the first ERC posed a significant risk factor, as well as aneuploidy at some point during the follow-up period $(p<0.001-0.005)$. We did not analyse aneuploidy further, since we only had data on this variable for a portion of our patients. In multivariate analysis we found a suspicious BC burden over 0.05, CEA>ULN burden and GGT burden represented significant independent risk factors for $\mathrm{CCA}(\mathrm{p}<0.001, p<0.001$, and $p=0.005$, respectively). Corresponding analysis for neoplasia (dysplasia or CCA) can be found in Supplementary Table I.

\section{Discussion}

In this large single centre study including 447 non-selected PSC patients with 788 ERCs and BCs with a median follow-up of 6 years, CCA was detected in 17 (3.8\%) patients, and a non-malignant course of disease, including biliary dysplasia (5.1\%), was seen in 424 patients. LT was performed in 46 patients. The sensitivity of suspicious/malignant BC was 71 to $100 \%$ with specificity of 91 to $93 \%$ in detecting CCA. To our knowledge, this is the first study evaluating the role of regular surveillance among non-selected PSC patients with 
ERC, using detailed ERC scoring, systematic BC, presence of inflammation in $\mathrm{BC}$, and laboratory values in relation to the risk of CCA.

Essential risk factors for CCA and dysplasia included advanced ERC changes, male gender, elevated ALT, AST, GGT, ALP, bilirubin, CA19-9 or CEA, suspicious BC, inflammation in $\mathrm{BC}$, and male gender. The presence of IBD did not correlate with biliary neoplasia, which is in line with some previous results (17), but somewhat differs from a US-based study (18). CA 19-9 is often elevated with CCA, but in PSC, up to one-third of patients may have an elevated CA 19-9 in the absence of CCA $(16,19)$. The AASLD Guidelines for PSC (20) do not recommend screening for CCA based on cytology. Our protocol differs from these guidelines. Based on the present study, individual risk stratification and targeting screening for the patients with highest CCA risk could be possible, if early liver transplantation or other early treatment is an option.

In an earlier study conducted in our hospital (9), ploidy analysis increased the sensitivity in the detection of biliary neoplasia. The material in the previous study, however, consisted of selected patients with a more advanced stage of PSC. This study then challenges the additional benefit of ploidy analysis among non-selected PSC patients due to the solid sensitivity of $\mathrm{BC}$ alone. The lack of additional advantage of ploidy analysis was also seen in another study among patients with newly diagnosed PSC (15). Also, the BC samples at the present study with the new collection technique were more representative containing an abundance of cells.

Inflammation in BC turned out to be a significant risk factor for CCA in this study. Cyclooxygenase-2 and microsomal prostaglandin E synthase-1 expression in nonneoplastic and CCA tissue is also up-regulated in PSC patients, emphasising the importance of inflammation in the carcinogenesis of PSC patients (21). Neutrophilic inflammation is easily graded in $\mathrm{BC}$, and, according to our study, stands as a significant co-factor with ERC 
changes for biliary neoplasia. We used inflammation burden in BCs in statistical analysis to evaluate the effect of continuous inflammation as a risk factor for CCA or dysplasia. It is, however, to be noted that inflammation in BC was common, and only $7.5 \%(11 / 146)$ of patients having purulent inflammation in the first ERC of the study period were later diagnosed with CCA. On the other hand, CCA was only seen in one patient with no inflammation burden in BC, patient having only one $\mathrm{BC}$ available during the study period. Inflammation in the colon biopsies of IBD patients predicts the risk of colorectal carcinoma and the use of immune modulators seems to reduce the risk $(22,23)$. The same is most likely to be true also with PSC. Using a combination of UDCA and metronidazole to reduce the inflammation of the bile ducts is reflected by decrease of ALP levels (24). The use of UDCA was not possible to include into statistical analysis, since $93 \%$ of patients were on UDCA therapy and it was not randomised.

Our aim was to determine the risk factors for CCA to target the surveillance of PSC patients better. The question remains, if prevention of CCA with liver transplantation is possible with systematic surveillance using ERC with BC. Low-grade dysplasia is a strong predictor of high-grade dysplasia in the bile ducts (6), but the natural course of dysplasia is not well characterized. Cytogenetic abnormalities similar to CCA are detected in low-grade dysplasia although less commonly than in high-grade dysplasia (25). Since CCA is generally regarded as a contraindication for LT, we were probably able to prevent several CCAs in this group of patients through systematic screening and early referral for liver transplantation. However, not all liver transplantations were performed due to neoplasia suspicion, but due to endstage liver disease or clinical symptoms. Still, 12 patients developed CCA more than one year after their PSC diagnosis, seven (58.3\%) of them presenting with suspicious/malignant BC. 
$B C$ interpretation is always subjective due to lack of objective markers. The number of BCs and the time between $\mathrm{BCs}$ varied considerably between patients, which might represent a weakness of this study. This was, however, taken into consideration in our statistical analysis.

Although ERC is an invasive method, our complication rate is acceptably low. In an earlier study from our unit, including 441 ERCs of PSC patients during 2007-2009, post-ERC pancreatitis was seen in 31 patients $(7.0 \%)$, of which three severe cases. All were treated conservatively (26). Cholangitis after ERC was seen 6 patients (1.4\%). This is comparable to previous studies (27). Considering the high risk of CCA in PSC patients, and the fact that PSC patients are often young, we find this complication rate acceptable. In patients with advanced age or other serious illnesses, ERC surveillance is not indicated, if the patients are not candidates for liver transplantation due to concomitant diseases. On the other hand, ERC with dilatation and stent placement if needed for dominant strictures in PSC is recommended in order to increase patients' survival (28). Dilatation and stenting is widely used for this purpose in our unit, and BC is regularly taken subsequently with dilatation to find a possible malignancy associated with strictures. Dominant stricture in our scoring equals to mERC score $\geq 3$ for extrahepatic bile ducts. However, not all CCAs can be prevented by ERC surveillance and liver transplantation. In ulcerative colitis, endoscopic surveillance is routinely performed because of the elevated colorectal cancer risk of 2.4-fold (29), whereas the risk of CCA in PSC patients is much more significantly increased, with the lifetime risk reaching almost $10 \%$ (1), or 398-fold compared to that for general population (2). Still, most centres carry no systematic surveillance for biliary dysplasia. It is debatable if the risk of complications of ERC and liver transplantation are acceptable. Only patients with repeatedly suspicious BC and the highest risk for CCA are considered for early liver transplantation. In addition, ERCs for PSC patients are performed by very experienced 
gastroenterologists to minimize the risks of the procedure, and to obtain representative BCs in our unit.

In conclusion, in this large study of non-selected PSC patients, advanced ERC changes with neutrophilic inflammation in $B C$ along with suspicious $B C$, male gender, elevated AST, ALT, ALP, bilirubin, GGT, CA19-9, and CEA indicated a higher risk of CCA and dysplasia. ERC surveillance with $\mathrm{BC}$ might be most beneficial for patients with such findings and could possibly identify patients with risk of progression to CCA if early LT is possible.

Disclosures: The authors have no conflicts of interest to disclose.

Financial support: This study was financially supported by the Sigrid Jusélius Foundation, the Mary and Georg Ehrnrooth Foundation, and the Gastroenterological Research Foundation. State funding for university-level health research from the Helsinki University Hospital also supported this work.

Table I. Demographics.

Demographic characteristics of the whole study population in the beginning of surveillance according to the endpoint. 
Range Benign/dysplasia CCA P

$(96,2 \%)^{\star *}$

(3.8\%)

Patients, $\mathbf{n}(\mathbf{M} / \mathbf{F})$

Age at PSC diagnosis, years

10-69

$430(234 / 196)$

$17(13 / 4)$

0.085

Symptomatic (\%)

$36(27-48)$

$43(31-57)$

0.164

$17 \%$

$47 \%$

0.005

\section{ERC findings}

-Intrahepatic only, $\mathrm{n}(\%)$

-Intra+extrahepatic, n (\%)

-Intrahepatic mERC-score (IQR)

2-8

0-7

2-14

-Total mERC-score (IQR)

Liver transplantation ${ }^{*}, \mathbf{n}$

IBD, $\mathbf{n}(\%)^{*}$

UC/CD/Indeterminate, $n$

IBD duration, years (IQR)

IBD after PSC diagnosis, $\mathbf{n}$

Intestinal dysplasia ${ }^{*}, \mathbf{n}$

$\mathrm{CRC}^{*}, \mathrm{n}$

Colectomy ${ }^{*}, \mathbf{n}$

AlH, $n$

UDCA $^{*}, \mathbf{n}$

Metronidazole*, $n$

Abbreviations: AlH, autoimmune hepatitis; CCA, cholangiocarcinoma; CD, Crohn's disease; CRC, colorectal carcinoma; F, female; IBD, inflammatory bowel disease; Indeterminate, indeterminate colitis; IQR, interquartile range; M, male; mERC score, modified Amsterdam ERC score; UC, ulcerative colitis; UDCA, ursodeoxycholic acid

*at the end of surveillance

**including 3 patients with gallbladder carcinoma or carcinoma in situ and 3 patients with hepatocellular carcinoma 
$\mathrm{p}$ values calculated with Mann-Whitney $\mathrm{U}$ test for dichotomous variables, and with Fisher's exact test for continuous variables. Continuous results are show as median (IQR). 
Table II. Laboratory values.

Laboratory values at the beginning of systematic surveillance.

\begin{tabular}{cccc} 
Range & Benign/dysplasia & CCA & P \\
\cline { 2 - 3 } & $(96,2 \%)^{*}$ & $(3.8 \%)$ & \\
$93-174$ & $139(127-148)$ & $128(115-149)$ & 0.198 \\
$49-736$ & $249(207-298)$ & $269(216-329)$ & 0.293 \\
$23.8-78.0$ & $38.6(35.7-40.9)$ & $39.7(36.8-40.3)$ & 0.944
\end{tabular}

\begin{tabular}{|c|c|c|c|c|}
\hline Hb, g/L (IQR) & $93-174$ & $139(127-148)$ & $128(115-149)$ & 0.198 \\
\hline Platelets, x $10^{9}$ (IQR) & $49-736$ & 249 (207-298) & 269 (216-329) & 0.293 \\
\hline Albumin, g/L (IQR) & $23.8-78.0$ & $38.6(35.7-40.9)$ & $39.7(36.8-40.3)$ & 0.944 \\
\hline TT, \% (IQR) & $19-170$ & $108(92-124)$ & 107 (85-132) & 0.675 \\
\hline INR (IQR) & $0.8-2.4$ & $1.0(0.9-1.1)$ & $1.1(0.9-1.1)$ & 0.243 \\
\hline Bilirubin, $\mu \mathrm{mol} / \mathrm{L}$ (IQR) & $2-426$ & $11(8-17)$ & $29(13-117)$ & $<0.001$ \\
\hline ALP, U/L (IQR) & $12-1002$ & $116(85-181)$ & $244(154-385)$ & $<0.001$ \\
\hline GGT, U/L (IQR) & $10-1979$ & $76(28-215)$ & 237 (119-643) & 0.002 \\
\hline AST, U/L (IQR) & $13-276$ & $34(25-51)$ & $50(36-94)$ & 0.002 \\
\hline ALT, U/L (IQR) & $4-537$ & $37(22-69)$ & $55(40-134)$ & 0.021 \\
\hline CA19-9, kU/L (IQR) & $2-1035$ & $5(3-11)$ & $14(6-73)$ & 0.009 \\
\hline CEA, $\mu \mathrm{g} / \mathrm{L}(\mathrm{IQR})$ & $1-28$ & $1.4(1.0-2.3)$ & $2.8(1.2-10.1)$ & 0.014 \\
\hline IgG4, g/L (IQR) & $0.01-5.54$ & $0.47(0.22-0.94)$ & $0.98(0.45-1.18)$ & 0.159 \\
\hline CRP, mg/L (IQR) & $0-107$ & $3(3-5)$ & $3(3-11)$ & \\
\hline
\end{tabular}


Abbreviations: ALP, alkaline phosphatase; ALT, alanine aminotransferase; AST, aspartate aminotransferase; CA19-9, carbohydrate antigen 19-9; CEA, carcinoembryonic antigen, CRP, C-reactive protein; GGT, gamma-glutamyl transferase; Hb, haemoglobin; IgG4, immunoglobulin G4; INR, international normalized ratio; TT, thromboplastin time

*including 3 patients with gallbladder carcinoma or carcinoma in situ and 3 patients with hepatocellular carcinoma p-values calculated with the Mann-Whitney $U$ test. Results are shown as median (IQR). 
Table III. Sensitivity and specificity.

Sensitivity and specificity of BC and DNA flow cytometry in the detection of CCA or biliary neoplasia (CCA or dysplasia).

\begin{tabular}{lcccc}
\hline ALL PATIENTS & Histology or follow-up (n) & & 95\% Cl \\
& & & Sens: $71 \%$ & $44.04-89.69 \%$ \\
BC only ( $\mathbf{n}=\mathbf{4 4 7 )}$ & CCA & Benign/Dysplasia & Spec: $91 \%$ & $87.81-93.47 \%$ \\
BC suspicious/malignant & 12 & 39 & PPV: $24 \%$ & $12.79-37.49 \%$ \\
BC benign & 5 & 391 & NPV: $98 \%$ & $97.08-99.59 \%$
\end{tabular}

Sens: $71 \% \quad 44.04-89.69 \%$

BC or FC $(\mathbf{n = 2 5 0 )} \quad$ CCA Benign/dysplasia $\quad$ Spec: $81 \% \quad 75.49-85.93 \%$

$\begin{array}{lllll}\text { Suspicious or aneuploid } & 12 & 44 & \text { PPV: } 21 \% & 11.59-34.44 \%\end{array}$

$\begin{array}{llll}\text { Benign and diploid } & 5 & 189 & \text { NPV: } 97 \%\end{array}$

\section{SURVEILLANCE COHORT}

Sens: $100 \% \quad 63.06-100.0 \%$

\begin{tabular}{lcccc} 
BC only (n=262) & CCA & Benign/dysplasia & Spec: $93 \%$ & $88.56-95.44 \%$ \\
BC suspicious/malignant & 8 & 19 & PPV: $30 \%$ & $13.75-50.18 \%$ \\
BC benign & 0 & 235 & NPV: $100 \%$ & $98.44-100.0 \%$ \\
\hline & & & Sens: $100 \%$ & $63.06-100.0 \%$ \\
BC or FC $(\mathbf{n}=107)$ & CCA & Benign/dysplasia & Spec: $79 \%$ & $69.42-86.36 \%$
\end{tabular}




\begin{tabular}{lcccc} 
Suspicious or aneuploid & 8 & 21 & PPV: $28 \%$ & $12.73-47.24 \%$ \\
Benign and diploid & 0 & 78 & NPV: $100 \%$ & $95.38-100.0 \%$ \\
\hline ENDPOINT NEOPLASIA (CCA or biliary dysplasia, all patients)
\end{tabular}

Sens: $65 \% \quad 48.32-79.37 \%$

BC only ( $\mathbf{n}=\mathbf{4 4 7 )} \quad$ Neoplasia $\quad$ Benign $\quad$ Spec: $94 \% \quad 91.07-95.99 \%$

$\begin{array}{lllll}\text { BC suspicious } & 26 & 25 & \text { PPV: } 51 \% & 36.60-65.25 \%\end{array}$

$\begin{array}{lllll}\text { BC benign } & 14 & 382 & \text { NPV: } 96 \% & 94.14-98.05 \%\end{array}$

\section{Sens: $69 \% \quad 52.43-82.98 \%$}

BC or FC ( $\mathbf{n}=\mathbf{2 5 0}) \quad$ Neoplasia $\quad$ Benign $\quad$ Spec: $86 \% \quad 80.86-90.60 \%$

$\begin{array}{llll}\text { Suspicious or aneuploid } & 27 & 29 & \text { PPV: } 48 \%\end{array} \quad 34.66-61.97 \%$

$\begin{array}{lllll}\text { Benign and diploid } & 12 & 182 & \text { NPV: } 94 \% & 89.44-96.76 \%\end{array}$

Abbreviations: $\mathrm{BC}$, brush cytology; CCA, cholangiocarcinoma; $\mathrm{Cl}$, confidence interval; FC, DNA flow cytometry; NPV, negative predictive value; PPV, positive predictive value; Sens, sensitivity; Spec, specificity 
Table IV. Risk factors.

Univariate and multivariate analyses of risk factors for CCA.

\section{Univariate}

Variable

Age at beginning of follow-up (years)

Sex (male/female)

Inflammation burden in BC

Extrahepatic mERC score burden

Intrahepatic mERC score burden

Total mERC score burden

mERC score burden $>4$

mERC score burden $>8$

ALT burden

AST burden

GGT burden

Bil burden

Bil >ULN burden

ALP burden

CEA > ULN burden

CA19-9 > ULN burden

Time from diagnosis to start of follow-up

Suspicious $B C$ at first ERC

Suspicious BC burden

Suspicious BC burden $>0.05$

Aneuploidy some time during follow-up
HR

1.0

5.5

3.9

1.5

2.0

1.4

54.9

18.8

9.5

24.3

10.9

43.3

13.0

27.3

15.4

9.8

1.5

13.9

31.6

18.1

5.2
$95 \% \mathrm{Cl}$

lower upper P

$\begin{array}{lll}1.0 & 1.1 & 0.329\end{array}$

$1.2 \quad 24.3$

0.025

$\begin{array}{lll}2.0 & 7.8<0.001\end{array}$

$1.2 \quad 1.9<0.001$

$1.4 \quad 2.9<0.001$

$1.2 \quad 1.6<0.001$

$0.8 \quad 3592.3 \quad 0.061$

$4.2 \quad 83.5<0.001$

$2.5 \quad 36.6 \quad<0.001$

$5.3 \quad 111.3<0.001$

$4.1 \quad 29.0 \quad<0.001$

$15.5 \quad 120.9<0.001$

$4.1 \quad 40.7<0.001$

$\begin{array}{lll}5.3 & 139.9<0.001\end{array}$

$4.8 \quad 49.1<0.001$

$3.5 \quad 28.1<0.001$

$\begin{array}{lll}0.8 & 2.7 & 0.197\end{array}$

$\begin{array}{lll}5.0 & 38.4<0.001\end{array}$

$11.0 \quad 90.9<0.001$

$6.4 \quad 51.4<0.001$

$\begin{array}{lll}1.6 & 16.3 \quad 0.005\end{array}$

\section{Multivariate}

GGT burden

$\begin{array}{llll}9.3(1.8) & 1.9 & 44.0 & 0.005\end{array}$


Bil > ULN burden

CEA > ULN burden

Suspicious BC burden $>0.05$

$3.1(2.0)$

0.8

12.3

0.101

$22.1(6.3)$

5.2

93.2

$<0.001$

$18.8(6.0)$

5.1

68.5

$<0.001$

Abbreviations: ALP, alkaline phosphatase; ALT, alanine aminotransferase; AST, aspartate aminotransferase; BC, brush cytology; Bil, bilirubin; CA19-9, carbohydrate antigen 19-9; CEA, carcinoembryonic antigen; GGT, gamma-glutamyl transferase; mERC score, modified Amsterdam ERC score; ULN, upper limit of normal value

Logarithmic transformation was used in continuous ALP, AST, ALT, GGT, CA 19-9, and CEA variables. The following variables were used for parameter selection into the multivariate model: sex, age, mERC score (total), suspicious/malignant brush cytology>0.05, ALP>ULN, ALT, GGT, CA199>ULN, CEA $>$ ULN, bilirubin $>U L N$, Inflammation burden in $\mathrm{BC}>0.65$ and time from diagnosis to start of follow-up. Nonzero HRs from the penalized (lasso) Cox model are shown in parentheses; other values are from unpenalized Cox models fitted with the parameters stated in the table.

FIGURE LEGENDS 
Figure 1. Surveillance protocol for PSC patients at Helsinki University Hospital.

Abbreviations: AMA, antimitochondrial antibody; CT, computed tomography; IBD, inflammatory bowel disease; mERC score, modified Amsterdam ERC score; MRI, magnetic resonance imaging; US, ultrasound

Figure 2. Outcome of the patients in the surveillance cohort. Abbreviations: BC, brush cytology; CCA, cholangiocarcinoma 
1. Boberg KM, Lind GE. Primary sclerosing cholangitis and malignancy. Best Pract Res Clin Gastroenterol 2011;25:753-764.

2. Boonstra K, Weersma RK, van Erpecum KJ, Rauws EA, Spanier BW, Poen AC, et al. Population-based epidemiology, malignancy risk, and outcome of primary sclerosing cholangitis. Hepatology 2013;58:2045-2055.

3. Boberg KM, Bergquist A, Mitchell S, Pares A, Rosina F, Broomé U, et al. Cholangiocarcinoma in primary sclerosing cholangitis: risk factors and clinical presentation. Scand J Gastroenterol 2002;37:1205-1211.

4. Chapman MH, Webster GJ, Bannoo S, Johnson GJ, Wittmann J, Pereira SP. Cholangiocarcinoma and dominant strictures in patients with primary sclerosing cholangitis: a 25-year single-centre experience. Eur J Gastroenterol Hepatol 2012;24:1051-1058.

5. Razumilava N, Gores GJ, Lindor KD. Cancer surveillance in patients with primary sclerosing cholangitis. Hepatology 2011;54:1842-1852.

6. Lewis JT, Talwalkar JA, Rosen CB, Smyrk TC, Abraham SC. Precancerous bile duct pathology in end-stage primary sclerosing cholangitis, with and without cholangiocarcinoma. Am J Surg Pathol 2010;34:27-34.

7. European Association for the Study of the Liver. EASL Clinical Practice Guidelines: management of cholestatic liver diseases. J Hepatol 2009;51:237-267.

8. Boberg KM, Jebsen P, Clausen OP, Foss A, Aabakken L, Schrumpf E. Diagnostic benefit of biliary brush cytology in cholangiocarcinoma in primary sclerosing cholangitis. J Hepatol 2006;45:568-574. 
9. Halme L, Arola J, Numminen K, Krogerus L, Mäkisalo H, Färkkilä M. Biliary dysplasia in patients with primary sclerosing cholangitis: additional value of DNA ploidity. Liver Int 2012;32:783-789.

10. Trilianos P, Selaru F, Li Z, Gurakar A. Trends in Pre-Liver Transplant Screening for Cholangiocarcinoma among Patients with Primary Sclerosing Cholangitis. Digestion 2014;89:165-173.

11. Trikudanathan G, Navaneethan U, Njei B, Vargo JJ, Parsi MA. Diagnostic yield of bile duct brushings for cholangiocarcinoma in primary sclerosing cholangitis: a systematic review and meta-analysis. Gastrointest Endosc 2014;79:783-789.

12. Aishima S, Kubo Y, Tanaka Y, Oda Y. Histological features of precancerous and early cancerous lesions of biliary tract carcinoma. J Hepatobiliary Pancreat Sci $2014 ; 21: 448-452$.

13. Barr Fritcher EG, Kipp BR, Halling KC, Clayton AC. FISHing for pancreatobiliary tract malignancy in endoscopic brushings enhances the sensitivity of routine cytology. Cytopathology 2014;25:288-301.

14. Lindberg B, Arnelo U, Bergquist A, Thörne A, Hjerpe A, Granqvist S, et al. Diagnosis of biliary strictures in conjunction with endoscopic retrograde cholangiopancreaticography, with special reference to patients with primary sclerosing cholangitis. Endoscopy 2002;34:909-916.

15. Boyd S, Tenca A, Jokelainen K, Mustonen H, Krogerus L, Arola J, et al. Screening primary sclerosing cholangitis and biliary dysplasia with endoscopic retrograde cholangiography and brush cytology: risk factors for biliary neoplasia. Endoscopy 2016,DOI http://dx.doi.org/10.1055/s-0041-110792 
16. Sinakos E, Saenger AK, Keach J, Kim WR, Lindor KD. Many patients with primary sclerosing cholangitis and increased serum levels of carbohydrate antigen 19-9 do not have cholangiocarcinoma. Clin Gastroenterol Hepatol 2011;9:434-439.

17. Yanai H, Matalon S, Rosenblatt A, Awadie H, Berdichevski T, Snir Y, et al. Prognosis of Primary Sclerosing Cholangitis in Israel is Independent of Coexisting Inflammatory Bowel Disease. J Crohns Colitis 2015;9:177-184.

18. Navaneethan U, Venkatesh PG, Jegadeesan R, Lourdusamy V, Hammel JP, Kiran $\mathrm{RP}$, et al. Comparison of outcomes for patients with primary sclerosing cholangitis associated with ulcerative colitis and Crohn's disease. Gastroenterol Rep 2016;4:4349.

19. Venkatesh PG, Navaneethan U, Shen B, McCullough AJ. Increased serum levels of carbohydrate antigen 19-9 and outcomes in primary sclerosing cholangitis patients without cholangiocarcinoma. Dig Dis Sci 2013;58:850-857.

20. Chapman R, Fevery J, Kalloo A, Nagorney DM, Boberg KM, Shneider B, et al. Diagnosis and management of primary sclerosing cholangitis. Hepatology 2010;51:660-678.

21. Ishii Y, Sasaki T, Serikawa M, Minami T, Okazaki A, Yukutake M, et al. Elevated expression of cyclooxygenase-2 and microsomal prostaglandin E synthase-1 in primary sclerosing cholangitis: implications for cholangiocarcinogenesis. Int $\mathrm{J}$ Oncol 2013;43:1073-1079.

22. Rubin DT, Huo D, Kinnucan JA, Sedrak MS, McCullom NE, Bunnaq AP, et al. Inflammation is an independent risk factor for colonic neoplasia in patients with ulcerative colitis: a case-control study. Clin Gastroenterol Hepatol 2013;11:16011608. 
23. Nieminen U, Jussila A, Nordling $S$, Mustonen $H$, Färkkilä MA. Inflammation and disease duration have a cumulative effect on the risk of dysplasia and carcinoma in IBD: a case-control observational study based on registry data. Int $\mathrm{J}$ Cancer 2014;134:189-196.

24. Färkkilä $M$, Karvonen $A L$, Nurmi $H$, Nuutinen $H$, Taavitsainen $M$, Pikkarainen $P$, et al. Treatment of primary sclerosing cholangitis with a combination of metronidazole and ursodeoxycholic acid: a 3-year randomized placebo-controlled trial. Hepatology 2004;40:1379-1386.

25. Kerr SE, Barr Fritcher EG, Campion MB, Voss JS, Kipp BR, Halling KC, et al. Biliary dysplasia in primary sclerosing cholangitis harbors cytogenetic abnormalities similar to cholangiocarcinoma. Hum Pathol 2014;45:1797-1804.

26. Ismail S, Kylänpää L, Mustonen H, Halttunen J, Lindström O, Jokelainen K, et al. Risk factors for complications of ERCP in primary sclerosing cholangitis. Endoscopy 2012;44:1133-1138.

27. Bangarulingam SY, Gossard AA, Petersen BT, Ott BJ, Lindor KD. Complications of endoscopic retrograde cholangiopancreatography in primary sclerosing cholangitis. Am J Gastroenterol 2009:104;855-860.

28. Thosani N, Banerjee S. Endoscopic retrograde cholangiopancreatography for primary sclerosing cholangitis. Clin Liver Dis 2014:18;899-911.

29. Jess T, Rungoe C, Peyrin-Biroulet L. Risk of colorectal cancer in patients with ulcerative colitis: a meta-analysis of population-based cohort studies. Clin Gastroenterol Hepatol 2012:10;639-645. 